\title{
Oclusión terapéutica. Desde las escuelas de oclusión a la Odontología Basada en Evidencia
}

\section{Therapeutic occlusion. From occlusal schools of thought to Evidence-based Dentistry}

\author{
Firmani $\mathrm{M}^{1}$, Becerra $\mathrm{N}^{2}$, Sotomayor $\mathrm{C}^{2}$, Flores $\mathrm{G}^{3}$, Salinas $\mathrm{JC}^{3}$
}

\begin{abstract}
RESUMEN
Tanto en la práctica clínica como en la academia, siempre está presente la interrogante de cuál es la mejor posición mandibular para organizar una oclusión terapéutica y satisfacer los requerimientos estéticos y funcionales de los pacientes. Esta pregunta se podría responder desde la perspectiva de las diferentes escuelas de oclusión, siguiendo sus técnicas y utilizando su aparatología. Sin embargo, debido a que no existe suficiente evidencia científica que sustente la superioridad de una escuela sobre la otra, es preciso hacer una revisión donde se expongan los principios en que se basan las distintas filosofías oclusales, los autores que les dieron origen, la época en que surgieron y la terminología que usaron -desde el siglo pasado hasta nuestros días-, contrastándolas con la literatura científica actual. Esta no es una tarea fácil, ya que los estudios disponibles acerca de oclusión presentan gran variabilidad en la definición operacional de los términos, haciendo difícil su comparación. Se sugiere para el futuro buscar una estandarización de las definiciones y técnicas de registro, con el objeto de generar evidencia científica confiable, conducente a la creación de protocolos de recomendación para la toma de decisiones clínicas predecibles desde el punto de vista oclusal.
\end{abstract}

Rev. Clin. Periodoncia Implantol. Rehabil. Oral Vol. 6(2); 90-95, 2013.

Palabras clave: Oclusión, escuelas de oclusión, filosofías oclusales, Odontología Basada en Evidencia.

\begin{abstract}
Both in clinical practice and academia, there is always the question about what is the best mandibular position to organize a therapeutic occlusion that satisfies functional and aesthetic requirements from patients. This question could be answered from the point of view of different occlusion schools of thought, following their techniques and using their apparatuses. However, due to the lack of scientific evidence to support the pre-eminence of a particular school over the rest, it is necessary to go through the princip les that provide the foundations for the different occlusal philosophies: the authors who gave birth to each school of thought, the time in which they were formed and the terminology they used, from the early $20^{\text {th }}$ century to present times and making a comparison with current scientific literature. This is not an easy task, given that available studies about occlusion feature a great variability in the operational definition of terms, making comparison difficult. The article suggests that it would be necessary for the future to look for a standardization of recording definitions and techniques. The aim is to generate reliable scientific evidence for the creation of protocols of recommendation for predictable decision-making from an occlusal point of view.
\end{abstract}

Rev. Clin. Periodoncia Implantol. Rehabil. Oral Vol. 6(2); 90-95, 2013.

Key words: Occlusion, occlusion schools of thought, occlusal philosophies, Evidence-Based Dentistry.

\section{INTRODUCCIÓN}

La oclusión ha sido definida por Davis y Gray como el contacto existente entre los dientes ${ }^{(1)}$. Estos contactos pueden ser considerados tanto en estática, cuando los dientes contactan en máxima intercuspidación (MIC) al finalizar el cierre mandibular, como en dinámica, es decir, cuando los dientes se deslizan entre sí con el movimiento mandibular ${ }^{(1)}$. La definición de oclusión publicada por el Glosario de Términos Odontológicos, versión 8 (GTO-8), tiene dos acepciones: 1.- "El acto o proceso de cierre, o corte", y 2.- "La relación estática entre las superficies de corte o masticación de los dientes maxilares y mandibulares análogos". Además, se define oclusión funcional como: "Los contactos entre los dientes maxilares y mandibulares durante la masticación y deglución"(2,3). La oclusión forma parte del Sistema Masticatorio o Estomatognático (SE), compuesto por el ligamento periodontal, las articulaciones temporomandibulares (ATMs), los músculos de la masticación, sus tendones y ligamentos. Todo lo anterior es controlado por elementos neurales a través de impulsos aferentes provenientes del sistema nervioso central, a cargo de centros corticales y subcorticales que generan patrones centrales modulados por los receptores del sistema nervioso periférico desde las estructuras orales, articulares y musculares ${ }^{(4,5,11,12)}$. Una oclusión alterada o inestable podría tener un rol en el desarrollo de trastornos temporomandibulares (TTM), aunque la literatura actual no es concluyente al respecto(6-10,12). Por otra parte, estaría asociada a trastornos posturales, fonoarticulatorios, a falta de estética ${ }^{(1,13)}$ e incluso a cambios en las cargas a nivel plantar durante la marcha ${ }^{(14,72)}$. Se postula además que la masticación tiene un importante rol en la integración somatosensorial cerebral para mantener las funciones de aprendizaje y memoria del hipocampo, ayudando a controlar disfunciones cognitivas ${ }^{(15)}$.

La realización de rehabilitaciones orales complejas requiere asumir directrices basadas en conceptos que lleven al logro de un resultado óptimo para poder predecir el comportamiento de

1. Instructora. Departamento de Prótesis, Área de Oclusión. Universidad de Chile. Chile.

2. Ayudante. Departamento de Prótesis, Área de Oclusión. Universidad de Chile. Chile.

3. Profesor Asistente. Departamento de Prótesis, Área de Oclusión. Universidad de Chile. Chile.

Correspondencia autor: Mónica Firmani Villarroel. mfirmani@odontologia.uchile.cl. Trabajo recibido el 16/12/2011. Aprobado para su publicación el 01/02/2012. 
restauraciones y prótesis en boca, asegurando permanencia en el tiempo, estabilidad mandibular, comodidad para el paciente y evitando iatrogenias. Este paradigma ha promovido el surgimiento y sucesión de distintas escuelas de pensamiento en oclusión, que van desde aquellas basadas en principios de ingeniería y mecánica de la mandíbula, hasta conceptos de oclusión natural y terapéutica basados en principios biológicos. Por otra parte, ante el reconocimiento de que factores oclusales tienen cada vez menos relevancia en la etiología de los TTM, se ha ampliado la mirada hacia un manejo más integral, siendo la terapia oclusal una más ${ }^{(1,7,8,10,11,12,16-21)}$.

El propósito de este artículo es realizar una revisión del concepto de oclusión, su importancia clínica y fundamentos, desde el prisma de las distintas filosofías oclusales contrastadas con la Odontología Basada en Evidencia (OBE).

\section{LA OCLUSIÓN DENTARIA}

La Organización Mundial de la Salud (OMS) determinó en el año 2000 que el objetivo terapéutico en salud oral debe ser la mantención de al menos 20 dientes bien distribuidos estética y funcionalmente, que no requieran prótesis, permitiendo una dentición estable en el tiempo, donde las funciones orales estén satisfechas para la mayoría de la población ${ }^{(22)}$. Sin embargo, estudios indican que la estabilidad oclusal se ve comprometida con la falta de piezas posteriores, sin olvidar que la decisión debiera tomarse de acuerdo a las demandas y necesidades de cada paciente en particular ${ }^{(23)}$.

Biológicamente, se considera a la oclusión como una herramienta del ser humano para asegurar la masticación, no solo por nutrición sino que para mantener las funciones físicas, mentales y de salud en general. Recientemente se ha sugerido que la masticación en adultos mayores estaría asociada a un mejor estatus cognitivo, ya que aumentaría la irrigación cerebral(22). Psicosocialmente involucra el concepto de habilidad social, se traduce en que la apariencia física o estética influye tanto en sus relaciones y habilidades interpersonales como en la satisfacción personal. Otras funciones incluyen la percepción táctil, la fonética y el gusto(1,12,22,23).

La preservación de la dentición, y por lo tanto de la dimensión vertical del tercio inferior del rostro, permite a largo plazo una adecuada estabilidad oclusal, la cual mantiene las relaciones del resto de los componentes del $\mathrm{SE}^{(34,73)}$. Si se considera que cambios biomecánicos inducen una respuesta biológica en los distintos componentes del SE y por lo tanto en su respuesta adaptativa, se hace necesario plantear la búsqueda de objetivos que conduzcan a una buena práctica clínica en oclusión ${ }^{(1,7,12,22,24)}$.

\section{RELACIÓN CÉNTRICA Y OCLUSIÓN CÉNTRICA}

Dos de los términos más controversiales en oclusión son Relación Céntrica $(R C)$ y Oclusión Céntrica $(\mathrm{OC})$. El glosario de términos prostodónticos (GTO) lamentablemente ha contribuido a mantener esta confusión al cambiar sus definiciones varias veces a lo largo de los años. En 1956, publica que RC es "la relación más retruída (forzada) de la mandíbula con respecto al maxilar cuando los cóndilos están en la posición más posterior en la fosa glenoídea, a partir de la cual se realizan los movimientos laterales, a cualquier grado de separación mandibular". La OC es considerada una posición interdentaria. En 1987, publica la siguiente definición de RC: "La relación máxilomandibular en la cual los cóndilos están en la porción más anterosuperior (no forzada) enfrentando a la vertiente posterior de la eminencia con la porción más delgada y avascular del disco, es independiente del contacto dentario y se evidencia cuando se logra un movimiento rotatorio puro en el eje transversal". Además, cambia la definición de OC, definiéndola como "el contacto entre los dientes oponentes cuando la mandíbula está en RC y puede o no coincidir con máxima intercuspidación dentaria (MIC)". Este cambio de definición clínicamente se tradujo en una drástica disminución de la magnitud del deslizamiento en céntrica en la población y con ello la necesidad de realizar ajustes oclusales como indicación terapéutica. Lejos de contribuir a un mejor entendimiento entre especialistas, este cambio aumenta la confusión. En 1994, el GTO publica nuevamente las definiciones, manteniendo la RC como en 1987, pero aclaró la definición de OC como "la posición de máxima intercuspidación dentaria independiente de la posición condilar". Finalmente, en 2005, el GTO vuelve a cambiar la definición y determina que OC es "aquella oclusión coincidente con RC y puede o no coincidir con MIC"(2,3,25-29). Debido a estos cambios, el término OC quedó con esas dos definiciones en uso hasta el día de hoy ${ }^{(11,30)}$. En este artículo se ha decidido usar el término OC como sinónimo de MIC y Oclusión en Céntrica cuando OC coincide con $\operatorname{RC}^{(1,2,6,12,25,27,28,31,32,33,70)}$.

Otros conceptos en uso son "Posición Músculo Esqueletal Estable (PMEE) de la Mandíbula", descrita por Okeson (2003) incorporando el componente anterosuperior de fuerza de los músculos elevadores a la definición de RC antes citada(16,17), y "Centricidad Mandibular" (CM) descrita en Chile por Rodríguez (1985) como la disposición de la mandíbula en relación al macizo craneofacial, en la cual los cóndilos están ubicados anterosuperiormente en la cavidad glenoidea, enfrentando la pared posterior de la eminencia articular con el disco interpuesto en su porción más delgada y medialmente en relación al plano medio sagital de un paciente dado. La CM es mantenida por la función estabilizadora de la neuromusculatura masticatoria en estado de salud y equilibrio, dándose las óptimas condiciones neurofisiológicas, vasculares y metabólicas que preserven al SE frente a sus requerimientos funcionales. La CM se da en un sistema biológico, lo que implica que existe un rango fisiológico representado por una pequeña área de centricidad $^{(34)}$

\section{OCLUSIÓN TERAPÉUTICA Y ESCUELAS DE OCLUSIÓN}

Para reconstruir una dentición en pacientes desdentados completos, inicialmente se usaron registros de cera interoclusales donde el paciente cerraba en mordida habitual; el problema fue que eran muy inexactos. Posteriormente, se desarrollaron los registros gráficos de arco gótico, donde el paciente tenía que llevar la mandíbula lo más atrás posible, mas los registros no eran reproducibles y ni estables $^{(25)}$. En esos años aparecen las ideas que se transformaron en filosofías oclusales permitiendo el surgimiento de las escuelas de oclusión, cuyos fundadores no eran científicos, sino más bien clínicos inquietos que basándose en sus observaciones empíricas, definieron la posición condilar en la cavidad glenoídea como la principal determinante oclusal.

\section{ESCUELA GNATOLÓGICA}

Stallard fue el primero en usar el término Gnatología en 1924, definiéndola como la ciencia que estudia la anatomía, fisiología, histología y patología del SE. El GTO-8 (2005) actualiza esta definición relacionando el SE con la salud general del cuerpo, incluyendo el diagnóstico, la terapéutica y los procedimientos rehabilitadores $^{(2)}$. En 1926, McCollum funda la Sociedad Gnatológica, compartiendo con Harlam el crédito por inventar el método de registro del eje de bisagra a partir del uso del arco facial y trasladarlo a un articulador.

Stuart y McCollum publican en 1955 los principios del movimiento mandibular, del eje transversal de bisagra y de las relaciones máxilo mandibulares que debían ser transferidas a un articulador para reproducir el movimiento bordeante de la mandibula. Es interesante mencionar que McCollum creía que el principio de oclusión balanceada bilateral, útil en prótesis completas, era el esquema indicado para la restauración de la oclusión natural. Sin embargo, Stuart observó que estos contactos causaban interferencias que sacaban de posición céntrica a la mandíbula y los pacientes se quejaban ya que se mordían la lengua y mejillas, lo que lo lleva a replantear sus principios ${ }^{(12,25,26,35,36)}$.

Posteriormente, la Escuela Gnatológica postula que la 
mandíbula está en RC cuando los centros de movimiento vertical, transversal y horizontal están en su eje terminal de bisagra, posición que se logra cuando los cóndilos están en su posición más posterior, superior y medial en sus respectivas fosas (RUM: Rearmost, Uppermost, Midmost). Estos principios fueron apoyados en los ' 70 por los estudios radiológicos de Weinberg, basados en los cambios posicionales del cóndilo en la cavidad glenoidea que ocurren en OC. Este concepto cambió a partir de la década del ' 80 (pasando de la posición condilar más posterior a la más anterior, como aparece en la definición de RC del GTO-5 de 1987), así como los métodos clínicos para lograrla, siendo la técnica más aceptada la manipulación mandibular bimanual de Peter Dawson $(26,33,35)$

El concepto Gnatológico tiene tres requisitos para lograr una posición mandibular reproducible: deprogramar la musculatura, estabilizar las ATMs y eliminar los contactos deflectivos. Por lo tanto, es indispensable realizar registros pantográficos antes de reorganizar la oclusión hacia una oclusión orgánica, caracterizada por: Oclusión mutuamente protegida; guía canina; oclusión en céntrica en sentido puntiforme; contactos uniformes, simultáneos y simétricos en OC (twin centric contact); axialización de fuerzas a nivel dentario con contactos cúspide a fosa en tripoidismo; tabla oclusal estrecha; máxima altura cuspídea; y profundidad de fosas con anatomía suplementaria para lograr màxima eficiencia masticatoria. Además, se requiere de caras oclusales precisas, considerando el número de contactos interoclusales necesarios -187-, los cuales tienen que ocurrir simultáneamente en oclusión en céntrica ${ }^{(26,35)}$

Actualmente, los conceptos gnatológicos no sólo son usados como objetivo terapéutico en rehabilitación oral protésica, sino que son aplicados en ortodoncia para dentición natural.

Roth introdujo este pensamiento en los ' 70 , postulando además que el hecho de no lograr los principios gnatológicos en un paciente lo predispone al desarrollo de $\mathrm{TTM}^{(37)}$. Otra escuela derivada de la anterior es la Bioestética Dental, que comparte los principios gnatológicos y le suma lineamientos estéticos ${ }^{(38)}$.

\section{ESCUELA ESCANDINAVA O DE DESLIZAMIENTO EN CÉNTRICA}

En los países escandinavos, la oclusión fue un área de especial interés, siendo desarrollada por Arstad, Beyron, Brill, KroghPoulsen y Posselt, entre otros ${ }^{(35)}$. Ellos consideraban la oclusión como parte de un sistema relacionado con el macizo craneofacial que debía estar en armonía morfofuncional y apoyaban el concepto de área céntrica, a partir de estudios en jóvenes aparentemente normales, donde el $90 \%$ podía retruir su mandíbula en $1 \mathrm{~mm}$ posterior a OC. Una retrusión mayor -de 2 a $3 \mathrm{~mm}$-, aunque desfavorable, parecía ser bien tolerada si presentaba un deslizamiento en céntrica anteroposterior rectilíneo. Éste sólo se lograba por contactos dentarios bilaterales, de tal forma que los cóndilos funcionaran simétricamente, no así en el caso de una trayectoria lateral donde las condiciones serían desfavorables para funciones articular y muscular adecuadas. Estos autores defendían la idea de registrar la relación céntrica a través del eje de bisagra terminal que se da en el radio de la rotación mandibular pura, dentro de los 15 a $20 \mathrm{~mm}$ de distancia interincisal en apertura, utilizando para ello articuladores tipo no arcón. Las características oclusales deseables incluían: lograr una aceptable altura facial y distancia interincisal en reposo post-tratamiento; estabilidad mandibular en cierre con contactos bilaterales; distribución uniforme de contactos oclusales tanto en OC como en posición de contacto retrusiva (PCR); fuerzas axiales sobre cada diente; y movimientos de cierre y lateralidad sin interferencias, con la relación cóndilofosa y función muscular normales. Se establece que debe existir un deslizamiento en céntrica sagital menor o igual a $1 \mathrm{~mm}$ (entre 0.5 a 1 $\mathrm{mm}$ ) y el tejido blando no debe interferir durante el contacto dentario. Además, el tratamiento debe prevenir la generación de palancas en los dientes que puedan producir cambios posicionales en los mismos, restaurando puntos de contacto interproximales así como oclusales. Finalmente, en movimientos excursivos debe haber contactos de función de grupo en el lado de trabajo ${ }^{(7,8,39,40,41)}$.

Escuela Pankey, Mann y Schuyler (PMS), o de Libertad en Céntrica. Pankey y Mann (1950) promovieron una filosofía restauradora cuyo objetivo era lograr una oclusión balanceada bilateral, en base a un articulador llamado "P-M instrument". Este sistema estaba basado en la teoría esférica de Monson y en el registro de trayectorias oclusales funcionalmente generadas (FGP) de Meyer ${ }^{(25,36)}$. Estos conceptos fueron influenciados por Schuyler, quien fue el primero en hablar de Libertad en Céntrica(25).

Schuyler afirmaba que para evitar que el paciente protruyera la mandíbula al cerrar la boca, debía colocar la punta de la lengua en el paladar, centrando la mandíbula y usando una leve presión posterior en la manipulación mandibular para llegar a RC. En este concepto la $\mathrm{RC}$ coincide con $\mathrm{OC}$, pero en una área plana en la fosa central de los dientes posteriores sin influencia de la inclinación cuspídea, lo que permite un grado de libertad de las cúspides antagonistas a una misma dimensión vertical oclusal (DVO) ${ }^{(42)}$. Esto se logra mediante odontología restauradora o ajuste oclusal por desgaste selectivo en dentición natural y permite que la mandíbula cierre en su trayectoria habitual a OC, sin la respuesta de adaptación neuromuscular provocada por el contacto prematuro. Los rangos son diferentes para oclusión terapéutica y natural: en una terapéutica se acepta un área plana en sentido anterior y lateral de hasta $2 \mathrm{~mm}$, ya que esto permite disminuir el estrés funcional y podría favorecer la estabilidad de las rehabilitaciones protésicas; mientras tanto, el área de libertad en céntrica en oclusión natural es de 0.5 a $1 \mathrm{~mm}$ en ambos sentidos. El rango anteroposterior está dado sagitalmente por el contacto oclusal en RC hasta OC (céntrica larga) y el lateral está determinado por la trayectoria lateral de éste (céntrica ancha). Este concepto también se aplica en la estabilización de dispositivos interoclusales neuromusculares ${ }^{(27,33,35,42,43)}$. Ash y Ramfjord (1996) promovieron el concepto de oclusión óptima o ideal, que tenía como norte un ideal estético y fisiológico y que evolucionó hasta identificarse con la salud, comodidad y funcionalidad de la oclusión.

Sus principios eran la armonía del SE asegurada por el cumplimiento de los siguientes requisitos: estabilidad mandibular cuando los dientes contactan en RC; OC ligeramente anterior a RC, estrictamente en el sentido sagital (a nivel articular, esta distancia sería de 0.1 a $0.2 \mathrm{~mm}$ y a nivel dentario entre 0.2 y $0.5 \mathrm{~mm}$ ); excursiones en completa libertad con movimientos suaves de contacto oclusal, siendo éste mayor en el lado de trabajo que en el de no-trabajo; sin impacto ni desplazamiento de los dientes anteriores al cierre oclusal (frémito) y óptima dirección de fuerzas oclusales asegurando estabilidad dentaria. Esta oclusión ideal no debe indicarse en pacientes con oclusión natural funcionalmente sana(44).

\section{ESCUELA DE LA OCLUSIÓN NEUROMUSCULAR}

En las décadas de los '70 y '80 se desarrollaron muchos estudios para encontrar la posición más estable de la mandíbula, innovando en instrumentación clínica. Jankelson, Swain y Crane introdujeron un aparato electrónico llamado kinesiógrafo mandibular o myo-monitor, que mediante el uso de estimulación neural eléctrica transcutánea (TENS) aplicada en los nervios motores del trigémino y facial, tiene como función relajar la musculatura mandibular y facial para deprogramarla, identificando la posición de reposo verdadera. Posteriormente, monitoreando la posición mandibular con el Myo-monitor se podría estimular la musculatura para alcanzar el espacio interoclusal óptimo, determinando una correcta posición vertical funcional llamada posición miocéntrica (PMC), esto es, la posición mandibular óptima que relaciona la mandíbula con el cráneo cuando los dientes están en contacto $^{(25,27,28,45)}$. Debido a que las contracciones inducidas por el Myomonitor tienen la misma actividad química que la actividad muscular normal, se restauraría la fisiología muscular. Cuando la OC coincide con la PMC existe oclusión miocéntrica; si no hay coincidencia, la OC observada se llamará maloclusión miocéntrica. Estos conceptos se basan en la musculatura como factor dominante para generar posiciones mandibulares. Los movimientos mandibulares libres parten y vuelven desde la posición de reposo, que es individual para cada paciente, constituyéndose como el punto de partida más confiable para evaluar dichos movimientos. En la mayoría de los casos, la PMC es anterior a la $\mathrm{OC}$ del paciente, generando una interferencia en el sector anterior disminuyendo el overbite y causando una falta de soporte vertical 
posterior al quedar en inoclusión. Por lo tanto, una vez que la PMC es alcanzada, generalmente el caso es terminado con procedimientos ortodóncicos ${ }^{(45)}$.

\section{OCLUSIÓN CON ENFOQUE CONSERVADOR, BASADO EN LA OCLUSIÓN BIOLÓGICA}

El desarrollo de la dentición en humanos es considerado un proceso biológico complejo y continuo a lo largo de la vida, teniendo como determinantes las variables étnicas y la edad ${ }^{(46)}$. Conocida con el nombre de oclusión natural u oclusión biológica, presenta una gran variabilidad morfofuncional, manteniendo su condición fisiológica ${ }^{(47)}$. Por otro lado, la oclusión también se clasifica en oclusión fisiológica y oclusión patológica respectivamente según si está o no en armonía con los demás determinantes del movimiento mandibular, mientras que aquella que es capaz de producir cambios deletéreos en el SE, se denomina oclusión patogénica ${ }^{(19)}$. La oclusión terapéutica con enfoque conservador es aquella oclusión restaurada por el odontólogo, que en caso de haber armonía con las relaciones máxilo-mandibulares no interviene la relación de contactos dentarios pre-existentes, manteniendo la $\mathrm{OC}$ del paciente. Se justifica cuando el paciente tiene una oclusión óptima, cuando existe oclusión en céntrica, o cuando el paciente no presenta TTM $^{(48)}$.

\section{OCLUSIÓN Y ODONTOLOGÍA BASADA EN LA EVIDENCIA}

Para indagar acerca de la sustentabilidad de estos planteamientos, se realizó una búsqueda en Medline/Pubmed hasta julio 2011, asociando los términos "Dental Occlusion", "Philosophies" y "Evidence-Based Dentistry", además de una búsqueda manual con los términos "Esquemas Oclusales" y "Escuelas de Oclusión", en las referencias de los artículos revisados.

Los resultados de la búsqueda con los términos de "Dental Occlusion" arrojó un total de 21.018 artículos, al asociarlo con "Philosophies", baja a un total de 230 artículos los cuales se reducen a un total de 9 artículos si se asocia con "Evidence-Based Dentistry".

Esto indica la escasa información científica acerca del tema y la necesidad de hacer investigación que ponga a prueba los supuestos de cada filosofía oclusal(36).

La mayor parte de la literatura está basada principalmente en observaciones y teorías basadas en anécdotas empíricas más que en la prueba de hipótesis. Los estudios clásicos de Angle y Andrews establecieron los criterios de una oclusión óptima, basados principalmente en aspectos estéticos y anatómicos ${ }^{(3)}$. Posteriormente, Ash y Ramfjord señalaron que estos criterios poco tienen que ver con la estabilidad funcional y armonía neuromuscular en la que una oclusión ideal debe basarse ${ }^{(44)}$.

Morfológicamente, se ha asociado a determinados tipos de maloclusión con la presencia de TTM, tales como: mordida abierta, mordida profunda o cubierta, clases II y III de Angle, mordida cruzada, dientes inclinados, pérdida de molares, interferencias oclusales en el lado de no-trabajo y deslizamientos en céntrica mayores a $4 \mathrm{~mm}$. Se ha especulado que estas condiciones podrían ser la consecuencia de un TTM, más que su causa ${ }^{(10,47,61)}$. También, se ha asociado a la estructura esqueletal y forma facial con TTM, concluyendo que los adolescentes dólicofaciales (caras largas) y el tipo esqueletal de clase II tienen más signos y síntomas de TTM en una muestra dada(20,61). Troelzsch (2011) asocia la ocurrencia de cefaleas con un deslizamiento en céntrica mayor a $3 \mathrm{~mm}$. Estas conclusiones deben ser interpretadas con cuidado a la hora de extrapolar resultados ${ }^{(66)}$.

Rinchuse y Kandasamy (2006), basados en estudios en Resonancia Magnética, indican que a pesar de realizar correctamente las técnicas de obtención de RC, en un gran porcentaje los cóndilos no se encuentran en la supuesta posición de $\mathrm{RC}^{(32)}$. Dichos estudios sustentan lo postulado por Lindauer (1995), respecto a que durante el movimiento inicial de apertura y cierre no existiría un eje de rotación puro o eje terminal de bisagra, sino que es más bien un movimiento rototraslacional donde existen múltiples ejes de rotación instantáneos: la mandíbula rota y se traslada simultáneamente, en un movimiento condilar antero-posterior ${ }^{(51)}$. Finalmente, cuestionan la validez del uso del articulador como herramienta terapéutica confiable en todos los casos, dándole validez en aquellos donde se necesite realizar restauraciones complejas y cirugías ortognáticas, cuando la determinación de la DVO es crucial ${ }^{(32,37)}$. Clark (2001) recomienda el montaje en articulador en casos de deslizamientos en céntrica mayor a $2 \mathrm{~mm}$, de inestabilidad oclusal por pérdida de piezas dentarias, indicación de cirugía ortognática y por último, pre y post tratamiento ortodóncico, más por razones legales que científicas ${ }^{(50)}$.

Se ha responsabilizado a las interferencias oclusales y a la asimetría de contactos oclusales de causar movilidad dentaria, trauma oclusal, deflexión mandibular, bruxismo, migraciones dentarias y desbalance en la actividad muscular causando alteraciones en la función muscular, de las ATMs, y por consiguiente $\operatorname{TTM}^{(7,47,52,54,76)}$. Para responder a esto, Ciancaglini (2001) estudió la distribución de contactos oclusales en sujetos sanos y con síntomas de TTM y observó que la simetría en el número de contactos ocurre solamente en el $4 \%$ de los pacientes con TTM, respecto al $8 \%$ en sujetos sanos. La asimetría manifiesta (diferencia mayor a 3 contactos por lado) se encontró en un $72 \%$ de los sujetos con TTM y sólo en un $20 \%$ de los sujetos sanos ${ }^{(6)}$. En el año 2003, el mismo grupo de investigadores buscó la relación existente entre asimetría de contactos oclusales y TTM unilateral, concluyendo que hay una débil asociación. La ausencia de contactos bilaterales y simétricos parece ser la regla más que la excepción, tanto en sujetos sanos como con TTM, mostrando estos últimos mayor tasa de asimetría(53).

Por otro lado, se postula que los contactos oclusales pueden afectar la actividad electromiográfica (EMG) de los músculos masticadores ${ }^{(54)}$. Bakke y Moller (1980), provocaron un contacto prematuro artificialmente y observaron que la actividad EMG durante el máximo apriete voluntario (MAV) era asimétrica en todos los músculos, siendo mayor en el lado ipsilateral al contacto. Además, si este contacto era de mayor grosor, la actividad EMG descendía hasta ser similar en ambos lados, lo que se explica por una actividad reducida de los mecanoreceptores periodontales. Más recientemente, Li (2008) concluye que una interferencia unilateral de $0.5 \mathrm{~mm}$ aumenta la actividad EMG del temporal ipsilateral causando síntomas tipo cefaleas tensionales ${ }^{(75)}$. Ferrario (2002) observó que la actividad EMG durante MAV en adultos jóvenes con mayor cantidad de contactos oclusales ( $>$ a 10) era más alta que aquéllas con menor cantidad $^{(76)}$. Michelotti (2005) no encontró diferencias significativas en la actividad EMG en maseteros al introducir interferencias, postulando que sujetos sanos tienen mejor capacidad de adaptación a los cambios oclusales que sujetos que tienen o han presentado TTM(55).

Tartaglia (2008) comparó el comportamiento de la actividad EMG en músculos temporales, maseteros y esternocleidomastoídeos entre dentición natural y distintas rehabilitaciones orales, tanto con prótesis removible como con prótesis implanto-soportada, concluyendo que en apriete estático la actividad EMG sobre rehabilitaciones protésicas es similar a la dentición natural, observando un leve aumento en el temporal, pero que durante la masticación la coordinación neuromuscular era mayor en pacientes con dentición natural que en aquellos con rehabilitación protésica ${ }^{(57)}$.

La presencia de contactos de no-trabajo ha sido asociada como dañina en las ATMs ${ }^{(44)}$.

Okano (2005) observa que los contactos de trabajo reducen el desplazamiento condilar de trabajo y los contactos de no-trabajo reduce el desplazamiento condilar de no-trabajo ${ }^{(77)}$.

Seedorf (2009) va más allá y concluye que los contactos de no-trabajo podrían ser capaces de prevenir un potencial movimiento dañino superior de las ATMs ${ }^{(78)}$.

En cuanto al esquema oclusal en desoclusión, los estudios son controversiales a propósito de cuál es el más prevalente, concluyendo que ninguno predomina en la población, agregando que la presencia de contactos de no-trabajo parece ser la regla general entre la población ${ }^{(47,56)}$. Hay que distinguir que los contactos de no-trabajo son aquellos que no causan disfunción o daño en los componentes del SE, y las interferencias, ya sea en lado de trabajo, no-trabajo o de hiperbalance, son aquellos contactos que causan una desviación del patrón normal de movimiento mandibular, causando cargas potencialmente nocivas para el $\mathrm{SE}^{(56)}$.

En relación a la actividad EMG durante las excursiones 
laterales, se observó que en el lado de trabajo era mayor en músculos temporales anteriores y posteriores, pero si se presenta una interferencia oclusal de no-trabajo, la actividad EMG en temporales disminuía, mientras que no se observaron variaciones en la actividad EMG en maseteros ${ }^{(54)}$. Miralles $(2005,2007,2008)$ estudia la actividad EMG en maseteros y en músculos supra e infrahioideos, comparando desoclusiones laterales en guía canina y en función de grupo, demostrando que no había diferencias significativas en las tareas de deslizamiento desde céntrica hasta excéntrica (vis a vis dentaria), viceversa y apriete en excéntrica (vis a vis). Ambos esquemas tienen un efecto similar al evitar la excesiva actividad muscular durante las excursivas, señalando que la estabilidad mandibular en máximo apriete dentario es más importante que un esquema desoclusivo dado(58,59,60).

En relación a implantes y carga dentaria, la literatura indica que el éxito clínico y la longevidad del implante se pueden lograr a través de una oclusión controlada biomecánicamente. A diferencia de los dientes naturales, la sobrecarga o estrés en implantes se concentra en la cresta ósea marginal. Para evitar esto, hay que considerar la longitud del cantiléver, las parafunciones preexistentes y evitar los diseños oclusales inadecuados: tabla oclusal ancha, excesiva inclinación cuspídea y contactos prematuros e interferencias ${ }^{(23,62)}$. En este sentido, Shon (2011) concluye que se generan mayores fuerzas en el lado de trabajo en función de grupo completa que con guía canina y que se generan mayores fuerzas en el lado de notrabajo en balanceada bilateral(63). Lewis (2011), en una revisión sobre implantes unitarios, señala que no hay evidencia que un esquema oclusal sea superior a otro en cuanto a su comportamiento clínico(64). Wood y Vermilyea (2004) analizaron los factores de los posibles fracasos en restauraciones implanto soportadas, siendo la sobrecarga -especialmente la no axial- la responsable de la pérdida y/o fractura de los componentes de los implantes ${ }^{(65)}$. Las cargas laterales deben ser evitadas a través de contactos oclusales que permitan cargas axiales al implante. El esquema oclusal en implantes debe tender a evitar los contactos deflectivos, al igual que en dentición natural(36). En relación a los implantes, no hay evidencia científica que permita recomendar un tipo de esquema oclusal en particular(8).

En cuanto a prótesis completas, aun existe controversia con respecto a cuáles serían los factores oclusales más adecuados: el alineamiento dentario, forma dentaria o esquemas oclusales. Aunque los estudios no muestran diferencias significativas en cuanto a estos factores, la mayoría de los clínicos postula que debe haber contactos en todos los dientes posteriores en OC ${ }^{(7,74)}$. Farías (2010) comparó la eficiencia masticatoria en prótesis completas entre un esquema oclusal de guía canina y uno de balanceada bilateral, y no encontró diferencias estadísticamente significativas ${ }^{(52)}$.
En relación a pacientes ortodóncicos, Hudson (2011) cita a Okeson para señalar cuatro recomendaciones clínicas básicas: la oclusión en céntrica debe ser la posición mandibular de inicio de tratamiento; en lateralidad, debe haber un guía dentaria de contacto que permita una desoclusión inmediata del lado mediotrusivo (no-trabajo); la guía canina es la más recomendable; y en protrusiva, debe haber un esquema de mutua protección ${ }^{(67)}$.

En este sentido, Ash (2003-2007) promueve la idea que las interferencias oclusales deben evitarse incluso si están dentro de los límites de la capacidad adaptativa individual, agrega que en las universidades debiera enseñarse técnicas de ajuste oclusal conducentes a mejorar la estabilidad oclusal|(68,69).

\section{CONCLUSIONES}

Como conclusión, la elección de un esquema oclusal en cada paciente debiera considerar, de acuerdo al conocimiento actual, los patrones de masticación humanos, la morfología craneofacial y el tipo de oclusión estática previa, estableciendo así compatibilidades funcionales. Posiblemente, en sujetos con un patrón de masticación vertical calzaría mejor un esquema de guía canina, mientras que en aquellos con un patrón de masticación horizontal, un esquema con más libertad lateral consistente con una guía de función de grupo u oclusión balanceada(47). Concordamos con Davies (2001) quien enfatiza que antes de planficar un tratamiento se debe examinar el tipo de oclusión del paciente, su estado periodontal, ATMs, musculatura y relación con el componente craneocervical. Considerar además antecedentes de trastornos médicos, funcionales y de dolor orofacial. Debe hacerse un registro detallado de la oclusión inicial ya sea natural o intervenida, a través de fotografías, montaje de modelos o fichas clínicas, estableciendo un diagnóstico oclusal que permita comparar la oclusión que trae el paciente con la oclusión terapéutica que consideramos ideal para él en particular, según la mejor evidencia disponible ${ }^{(49,71)}$.

\section{CONFLICTOS DE INTERÉS}

Los autores declaran no tener conflictos de interés.

\section{REFERENCIAS BIBLIOGRÁFICAS}

1. Davies S, Gray R. What is occlusion? Brit Dent J, 2001; 191: 235-245.

2. The glossary of prosthodontic terms. J Prosthet Dent, 2005; 94: 21-22.

3. Clark JR, Evans RD. Functional occlusion: I a review. J Orthod, 2001; 28: 76-81.

4. Graf $H$, Geering $A H$. Rationale for critical application of differentes occlusal philosophies. Oral Sci Rev, 1977; 10: 1-10.

5. Forrester SE, Allen SJ, Fresswood RG, Toy AC, Pain MTG. Neuromuscular function in healthy occlusion. J Oral Rehabil, 2010; 37: 663-669.

6. Ciancaglini R, Gherlone EF, Redaelli S, Radaelli G. The distribution of occlusa contacts in the intercuspal position and temporomandibular disorder. J Oral Rehabil, 2002; 29: 1082-1090.

7. Carlsson GE. Some dogmas to prosthodontics, temporomandibular disorders and occlusion. Acta Odontol Scand, 2010; 68: 313-322.

8. Carlsson GE. Dental occlusion: Modern concepts and their application in implant prosthodontics. Odontology, 2009; 97: 8-17.

9. Miller JR. An endless discussion. Am J Orthod Dentofacial Orthop, 2011; 139: 293. 10. Mackie A, Lyons K. The role in temporomandibular disorders -a review of the literature. New Zealand Dental Journal, 2008; 104: 54-59.

11. Wilson PHR, Banerjee A. Recording the retruded contact position: A review of clinical techniques. Brit Dent J, 2004; 196: 395-402.

12. Türp JC, Greene CS, Strub JR. Dental occlusion: A critical reflection on past, present and future concepts. J Oral Rehabil, 2008; 35: 446-453.

13. Bracco P, Deregibus A, Piscetta R. Effects of different jaw relations on postural stability in human subjects. Neuroscience Letters, 2004; 356: 228-230.

14. Tecco S, Polimeni A, Festa F. Postural loads during walking after an imbalance of occlusion created with unilateral cotton rolls. BMC Res Notes, 2010; 3: 141.

15. Ono Y, Yamamoto T, Kubo L, Onozuka M. Occlusion and brain function: Mastication as a prevention of cognitive dysfunction. J Oral Rehabil, 2010; 8: 624-640.
16. Crawford SD. Condylar axis position, as determined by the occlusion and measured by the $\mathrm{CPI}$ instrument, and signs and symptoms of temporomandibular dysfunction. Angle Ortho, 1999; 69: 103-116.

17. Okeson JP. Tratamiento de oclusión y afecciones temporomandibulares. España: Elsevier, 2003.

18. Saizar P. La oclusión, diferentes escuelas. RAOA, 1979; 67: 113-120.

19. Racich MJ. Orofacial pain and occlusion: Is there a link? An overview of current concepts and the clinical implications. J Prosthet Dent, 2005; 93: 189196.

20. Gremillion HA. The relationship between occlusion and TMD: An evidencebased discussion. J Evid Base Dent Pract, 2006; 6: 43-47.

21. Davies SJ, Gray RM, Smith PW. Good occlusal practice in simple restorative dentistry. British Dental J, 2001; 191: 365-381.

22. Gotfretdsen K, Walls AWG. What dentition assures oral function? Clin Oral Imp/ Res, 2007; 18: 34-45.

23. Armellini D, Von Fraunhofer JA. The shortened dental arch: A review of the literature. J Prosthet Dent, 2004; 92: 531-535.

24. Tarantola GJ, Becker IM, Gremilion H. The reproductibility of centric relation: A clinical approach. J Am Dent Assoc, 1997; 128: 1245-1251.

25. Keshvad A, Winstanley RB. An appraisal of the literature on centric relation. Part I. J Oral Rehabil, 2000; 27: 823-833.

26. Pokorny $\mathrm{PH}$, Wiens JP, Litvak $\mathrm{H}$. Occlusion for fixed prosthodontics: A historical perspective of gnathological influence. J Prosthet Dent, 2008; 99: 299313

27. Keshvad A, Winstanley RB. An appraisal of the literature on centric relation. Part II. J Oral Rehabil, 2000; 27: 1013-1023.

28. Keshvad A, Winstanley RB. An appraisal of the literature on centric relation. Part III. J Oral Rehabil, 2000; 28: 55-63. 
29. Keshvad A, Winstanley RB. Comparison of the replicability of routinely used centric relation registration techniques. J Prosthodont, 2003; 12: 90-101.

30. Truitt J, Strauss R, Best A. Centric relation: A survey study to determine whether a consensus exists between oral and maxillofacial surgeons and orthodontists. $J$ Oral Maxillofac Surg, 2009; 67: 1058-1061.

31. Milosevic A. Occlusion I: Terms, mandibular movement and the factors of occlusion. Dent Update, 2003; 30: 359-361.

32. Rinchuse D, Kandasamy S. Centric relation: A historical and contemporary orthodontic perspective. JADA, 2006; 137: 494-501.

33. Becker CM, Kaiser DA, Schwalm C. Mandibular centricity: Centric relation. $J$ Prosthet Dent, 2000; 83: 158-160.

34. Rodríguez E. Centricidad mandibular. Rev Fac Odont Univ Chile, 1985; 3: 1.

35. Ash MM Jr. Philosophy of occlusion: Past and present. Dent Clin N Am, 1995; 39: 233-255.

36. Taylor TD, Wiens J, Carr A. Evidence-based considerations for removable prosthodontic and dental implant occlusion: A literature review. J Prosthet Dent, 2005; 94: 555-560.

37. Rinchuse DJ, Kandasamy S. Myths of orthodontic gnathology. Am J Orthod Dentofacial Orthop, 2009; 136: 322-330.

38. Benson JR. Exploring the world of bioaesthetic dentistry. Interview by Damon Adams. Dent Today, 2011; 98: 100-103.

39. Posselt U. Fisiología de la oclusión y rehabilitación. Barcelona: Jimbs, 1973. Reimpresión 1981.

40. Posselt U. Terminal hinge movement of the mandible. J Prosthet Dent, 1957; 7: $787-797$

41. Beyron H. Optimal occlusion. Dent Clin N Am, 1969; 13: 537-554.

42. Schuyler CH. Freedom in centric. Dent Clin N Am, 1969; 3: 681-686.

43. Starcke EN. The history of articulators: A critical history of articulators based on "geometric" theories of mandibular movement. Part IV: Needles, Wadsworth, and a look at some who followed. J Prosthodont, 2003; 12: 51-62.

44. Ash MM, Ramfjord S. Oclusión. México: McGraw Hill, 1996.

45. Dinham GA. Myocentric. A clinical appraisal. The Angle Orthodontist, 1984; 54: 211-217.

46. Thilander B. Dentoalveolar development in subjects with normal occlusion. A longitudinal study between the ages of 5 and 31 years. Eur J Orthodont, 2009; 31: 109-120.

47. Rinchuse DJ, Kandasamy S, Sciote J. A contemporary and evidence - based view of canine protected occlusion. Am J Orthod Dentofacial Orthop, 2007; 132: 90-102.

48. Davies SJ, Gray RMJ, Smith PW. Good occlusal practice in simple restorative dentistry. Br Dent J, 2001; 191: 535-581.

49. Davies SJ, Gray RMJ, Sandler PJ, O’Brien HD. Orthodontics and occlusion. Br Dent J, 2001; 191: 539-549.

50. Clark JR, Hutchinson I, Sandy JR. Functional occlusion: II. The role of articulators in orthodontics. J Orthod, 2001; 28: 173-177.

51. Lindauer SJ, Sabol J, Isaacson RJ, Davidovitch M. Condylar movement and mandibular rotation during jaw opening. Am J Orthod Dentofac, 1995; 107: 573 577

52. Farias A, Mestriner W, Carreiro A. Masticatory efficiency in denture wearers with bilateral balanced occlusion and canine guidance. Braz Dent J, 2010; 21: 165-169.

53. Ciancaglini R, Gherlone EF, Radaelli G. Unilateral temporomandibula disorder and asymmetry of occlusal contacts. J Prosthet Dent, 2003; 89: 180185.

54. Tovato F, Orlando B, Bosco M. Occlusal features and masticatory muscles activity. A review of electromyographic studies, Stomatologija. Baltic Dental and Maxillofacial Journal, 2009; 11: 26-31.

55. Michelotti A, Ferella M, Gallo LM, Veltri A, Palla S, Martina R. Effect of occlusal interference on habitual activity of human masseter. J Dent Res, 2005; 84: 644648.

56. Marklund S, Wänman A. A century of controversy regarding the benefit or detriment of occlusal contacts on the mediotrusive side. J Oral Rehabil, 2000; 27: 553-562.

57. Tartaglia GM, Testori T, Pallavera A, Marelli B, Sforza C. Electromyographic analysis of masticatory and neck muscles in subjects with natural dentition, teethsupported and implant-supported prostheses. Clin Oral Impl Rest, 2008; 19: 1081 1088.
58. Campillo MJ, Miralles R, Santander H, Valenzuela S, Fresno MJ, Fuentes A, Zúñiga C. Influence of laterotrusive occlusal scheme on bilateral masseter EMG activity during clenching and grinding. J Craniomandibular Pract, 2008; 4: 263-273. 59. Valenzuela S, Baeza M, Miralles R, Cavada G, Zúñiga C, Santande $\mathrm{H}$. Laterotrusive occlusal schemes and their effect on supra-and infrahyoid electromyographic activity. Angle Orthod, 2005; 76: 585-590.

60. Miralles R, Gallardo F, Baeza M, Valenzuela S, Ravera MJ, Ormeño G, Cavada G. Laterotrusive occlusal schemes and jaw posture tasks effects on supra-and infrahyoid EMG activity in the lateral decibitus position. J Craniomandibular Pract, 2007; 25: 106-113

61. Luther F. TMD and occlusion part I, damned if we do? Occlusion: The interface of dentistry and orthodontics. Br Dent J, 2007; 202: 1-7.

62. Kim Y, Oh T-J, Misch CE, Wang H-L. Occlusal considerations in implant therapy: Clinical guidelines with biomechanical rationale. Clin Oral Impl Res 2005; 16: 26-35

63. Sohn BS, Heo SJ, Koak JY, Kim SK, Lee SY. Strain of implants depending onocclusion types in mandibular implant-supported fixed prostheses. J Adv Prosthodont, 2011; 3: 1-9.

64. Lewis $\mathrm{M}$, Klineberg I. Prosthodontic considerations designed to optimize outcomes for single-tooth implants. A review of the literature. Aust Dent J, 2011; 56: 181-192.

65. Wood MR, Vermilyea SG. A review of selected dental literature on evidencebased treatment planning for dental implants: Report of the Committee on research in Fixed Prosthodontics of the Academy of Fixed Prosthodontics. J Prosthet Dent, 2004; 92: 447-462.

66. Troelzsch M, Troelzsch M, Cronin RJ, Brodine AH, Frankenberger R, Messlinger R. Prevalence and association of headaches, temporomandibular joint disorders, and occlusal interferences. J Prosthet Dent, 2011; 105: 410-417.

67. Hudson JM. Occlusal guidelines needed. Am J Orthod Dentofacial Orthop, 2011; 139: 425

68. Ash MM. Occlusion: Reflections on science and clinical reality. J Prosthet Dent 2003; 90: 373-384.

69. Ash MM. Occlusion, TMDs, and dental education. Head Face Med, 2007; 3: 1.

70. Christensen GJ. Is occlusion becoming more confusing? A plea for simplicity. JADA, 2004; 135: 767-770.

71. Davies SJ, Gray RMJ. The examination and recording of the occlusion: Why and how. Br Dent J, 2001; 191: 291-302.

72. Cuccia AM. Interrelationships between dental occlusion and plantar arch. JBMT, 2001; 15: 242-250.

73. Csadó K, Márton K, Kivovics P. Anatomical changes in the structure of the temporomandibular joint caused by complete edentulousness. Gerodontology, 2011; doi:10.111/j.1741-2358.2011.00498.x

74. Davies SJ, Gray RM, McCord JF. Good occlusal practice in removable prosthodontics. Br Dent J, 2001; 191: 491-502.

75. Li J, Jiang T, Feng H, Wang K, Zhang Z, Ishikawa T. The electromyographic activity of masseter and anterior temporalis during orofacial symptoms induced by experimental occlusal highspot. J Oral Rehabil, 2008; 35: 79-87.

76. Ferrario VF, Tartarglia GM, Galletta A, Grassi GP. The influence of occlusion on jaw and neck muscle activity: A surface EMG study in healthy young adults. J Ora Rehabil, 2006; 33: 341-348.

77. Okano N, Baba K, Ohyama T. The influence of altered occlusal guidance on condylar displacement during submaximal clenching. J Oral Rehabil, 2005; 32: 714 719

78. Seedorf H, Weitendorf H, Scholz A, Kirsch I, Heydecke G. Effect of non-working occlusal contacts on vertical condyle position. J Oral Rehabil, 2009; 36: 435-441. 